\title{
ROBOT HEXÁPODO CONTROLADO POR RADIO FRECUENCIA PARA LA BÚSQUEDA DE PERSONAS ATRAPADAS EN DERRUMBES EN ZONAS URBANAS DEL PERÚ
}

\section{RADIO FREQUENCY CONTROLLED HEXAPOD ROBOT TO SEARCH FOR PEOPLE TRAPPED IN LANDSLIDES IN URBAN AREAS OF PERU}

\author{
José C. Borja Padilla' y Ricardo J. Palomares Orihuela²
}

RECEPCIÓN: 30 DE OCTUBRE DE 2021

ACEPTACIÓN: 12 DE NOVIEMBRE DE 2021

\begin{abstract}
RESUMEN
La presente investigación presenta el diseño e implementación de un Robot hexápodo controlado por radiofrecuencia para la búsqueda de personas atrapadas en derrumbes en las zonas urbanas del Perú. El robot cuenta con $17 \mathrm{GDL}$, tiene como dimensiones $280 \mathrm{~mm}$ de alto y $560 \mathrm{~mm}$ de ancho, y fue sometido a cuatro pruebas. En la prueba 1, se verificó el análisis de estabilidad del robot. La prueba 2 entregó la respuesta del sistema de detección de temperatura de una persona con mediciones en el rango de 36.5 y $37^{\circ} \mathrm{C}$. En la prueba 3 , el robot, en un entorno en estado de derrumbe, realizó la búsqueda de personas en el área siniestrada. En la prueba 4, el robot, en un entorno cerrado y en estado de derrumbe, ingresó y permitió identificar una persona atrapada e iniciar un rescate seguro. Futuras investigaciones deben incluir un sistema autónomo de navegación y el uso de tecnologías loT.
\end{abstract}

Palabras clave: robot, hexápodo, búsqueda de personas, rescate.

\begin{abstract}
This research presents the Design and Implementation of a radio-frequency controlled hexapod Robot for the search of people trapped in landslides in urban areas of Peru. The robot has 17 DOF, its dimensions are 280 $\mathrm{mm}$ high and $560 \mathrm{~mm}$ wide, which was subjected to four tests. In test 1, the stability analysis of the robot was verified, test 2 gave the response of the temperature detection system of a person with measurements in the range of 36.5 and $37{ }^{\circ} \mathrm{C}$. In test 3 , the robot in an environment in a collapsed state carried out the search for people in the affected area, in test 4, the robot in a closed environment in a collapsed state, entered and allowed to identify a trapped person and initiate a safe rescue. Future research should include an autonomous navigation system and the use of loT technologies.
\end{abstract}

Keywords: robot, hexapod, search for people, rescue. 


\section{INTRODUCCIÓN}

En el Perú, durante los años 2017 y 2018, se registró un total de 418 sismos con una magnitud entre los 3.0 - 6.0 grados en la escala de Richter. El departamento de Arequipa presentó el más alto registro de eventos, 135, seguido por Lima con 45 eventos, Moquegua con 42 eventos e Ica con 32 eventos [1]. El mayor problema al ocurrir un desastre natural se relaciona con el Cuerpo General de Bomberos Voluntarios del Perú, que no cuenta con un sistema que les permita realizar la intervención de los rescatistas de manera óptima. Y es evidente que la disminución del tiempo de atención a los rescates influye de manera positiva en la preservación de la vida humana, tanto de las víctimas como de los rescatistas [2].

Debido a lo anterior, se realizó el diseño e implementación de un robot hexápodo con la capacidad para detectar la ubicación de víctimas atrapadas vivas, a través de la medición de su calor corporal, quienes permanecen atrapadas en espacios de difícil y peligroso acceso para los rescatistas, a partir de la ubicación de una zona segura de rescate.

\section{DISEÑO DEL ROBOT}

\subsection{Diseño mecánico}

Para el diseño mecánico del robot se utilizó la configuración de un robot móvil [3] del tipo zoomórfico - hexápodo con distribución radial. Además, se incluyeron dos brazos de 2 GDL, [4], [5], cada uno, para que pueda mover pequeńos obstáculos. El material seleccionado para el chasis fue el acero de carbono - manganeso GX120Mn13 [6]. Para sus dimensiones, se consideró las dimensiones de una persona de edad adulta en gateo. En la figura 1, se presenta el diseño de la estructura mecánica en el software de ingeniería Catia V5.

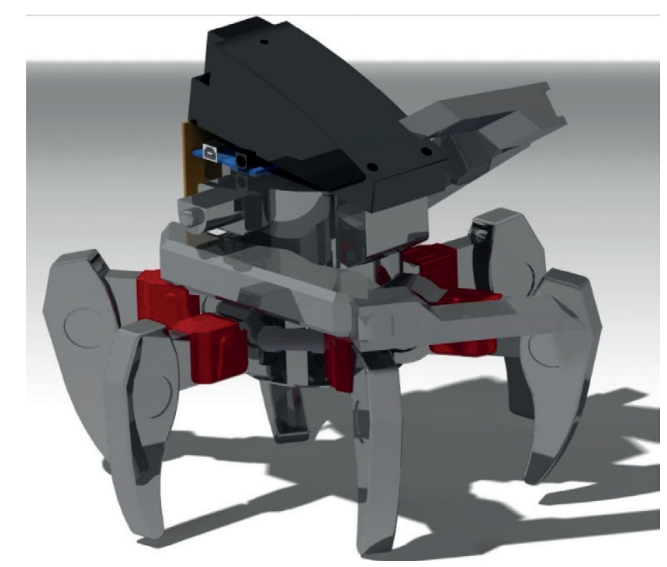

Figura 1. Diseño de la estructura mecánica mediante el software Catia V5. Elaboración propia.

\subsection{Diseño eléctrico}

Mediante la tabla 1, se presentan las características técnicas y la ubicación de los 03 tipos de micro servos que se utilizaron, con un voltaje de 12 Voltios, corriente de carga de 0.75 Amperios, potencia de $1.1 \mathrm{~W}$, así como un torque máximo de $1.8 \mathrm{~kg}-\mathrm{cm}$. 


\begin{tabular}{|c|c|c|c|c|c|c|c|}
\hline Nombre & $\begin{array}{c}\text { Voltaje } \\
(\mathrm{V})\end{array}$ & $\begin{array}{c}\text { Corriente } \\
\text { con carga } \\
\text { (A) }\end{array}$ & $\begin{array}{c}\text { Velocidad } \\
\text { sin carga } \\
(\mathrm{RPM})\end{array}$ & $\begin{array}{c}\text { Torque } \\
(\mathrm{kg}-\mathrm{cm})\end{array}$ & $\begin{array}{l}\text { Potencia } \\
\text { (W) }\end{array}$ & Cantidad & Ubicación \\
\hline Servo 1 & \multirow{3}{*}{12.0} & \multirow{3}{*}{0.75} & 210.0 & 1.8 & 1.0 & 1 & cuerpo \\
\hline Servo 2 & & & 320.0 & 1.3 & 1.1 & 6 & patas \\
\hline Servo 3 & & & 440.0 & 1.0 & 1.1 & 2 & brazos \\
\hline
\end{tabular}

Tabla 1. Micro servos utilizados en el robot hexápodo. Elaboración propia.

Se determinó el consumo de los componentes eléctricos y se obtuvo un consumo total de 10.73 Watts y un consumo total de la corriente de $850 \mathrm{mAmp}$, tal como se muestra en la tabla 2:

\begin{tabular}{|c|c|c|c|c|c|c|}
\hline $\mathrm{N}^{\circ}$ & Descripción & Cant. & $\begin{array}{c}\text { Consumo } \\
\text { Unitario } \\
\text { (Watts) }\end{array}$ & $\begin{array}{c}\text { Consumo } \\
\text { Total } \\
\text { (Watts) }\end{array}$ & $\begin{array}{l}\text { Consumo } \\
\text { mAmp }\end{array}$ & $\begin{array}{c}\text { Consumo } \\
\text { total x } 8 \text { horas } \\
\text { (Watts) }\end{array}$ \\
\hline 1 & Arduino UNO & 1 & 0.025 & 0.025 & 50 & 0.20 \\
\hline 2 & Servo 1 & 1 & 1.1 & 1.1 & 60 & 8.8 \\
\hline 3 & Servo 2 & 6 & 1.1 & 6.6 & 60 & 52.8 \\
\hline 4 & Servo 3 & 2 & 1.0 & 2.0 & 60 & 16.0 \\
\hline 5 & Módulo de Motor & 3 & 0.3 & 0.9 & 600 & 7.2 \\
\hline \multirow[t]{2}{*}{6} & Componentes varios & - & 0.1 & 0.1 & 20 & 0.8 \\
\hline & Total & & & 10.73 & 850 & 85.84 \\
\hline
\end{tabular}

Tabla 2. Cálculo de consumo eléctrico. Elaboración propia.

Mediante la figura 2, se presenta el esquema de distribución de energía del robot que consta de un (01) controlador Arduino UNO, tres (03) módulos para el motor y nueve (09) motores, de los cuales seis (06) permiten el control de las patas, dos (02) para el control de los brazos y uno (01) está ubicado en el cuerpo, a través del eje central del robot.

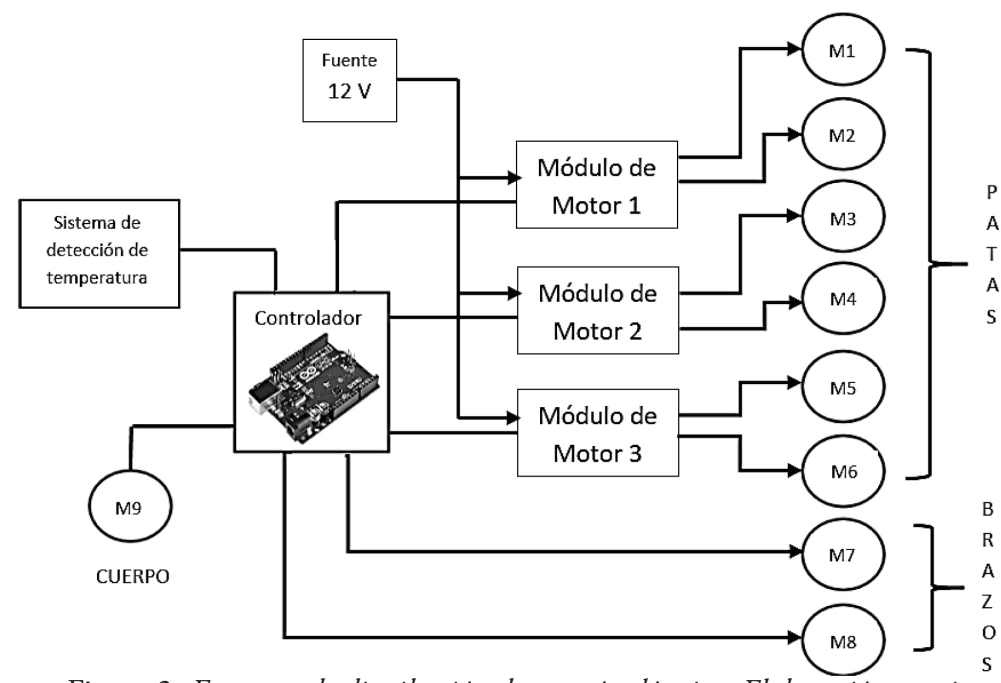

Figura 2. Esquema de distribución de energía eléctrica. Elaboración propia. 


\subsection{Diseño electrónico}

En la figura 3, se muestra el esquema electrónico del robot hexápodo diseñado en el software Proteus [7], que consta de un Controlador Arduino UNO, módulos para el motor de tipo L298 y 9 servo motores, de los cuales seis son para las patas, dos para los brazos y uno para el cuerpo.

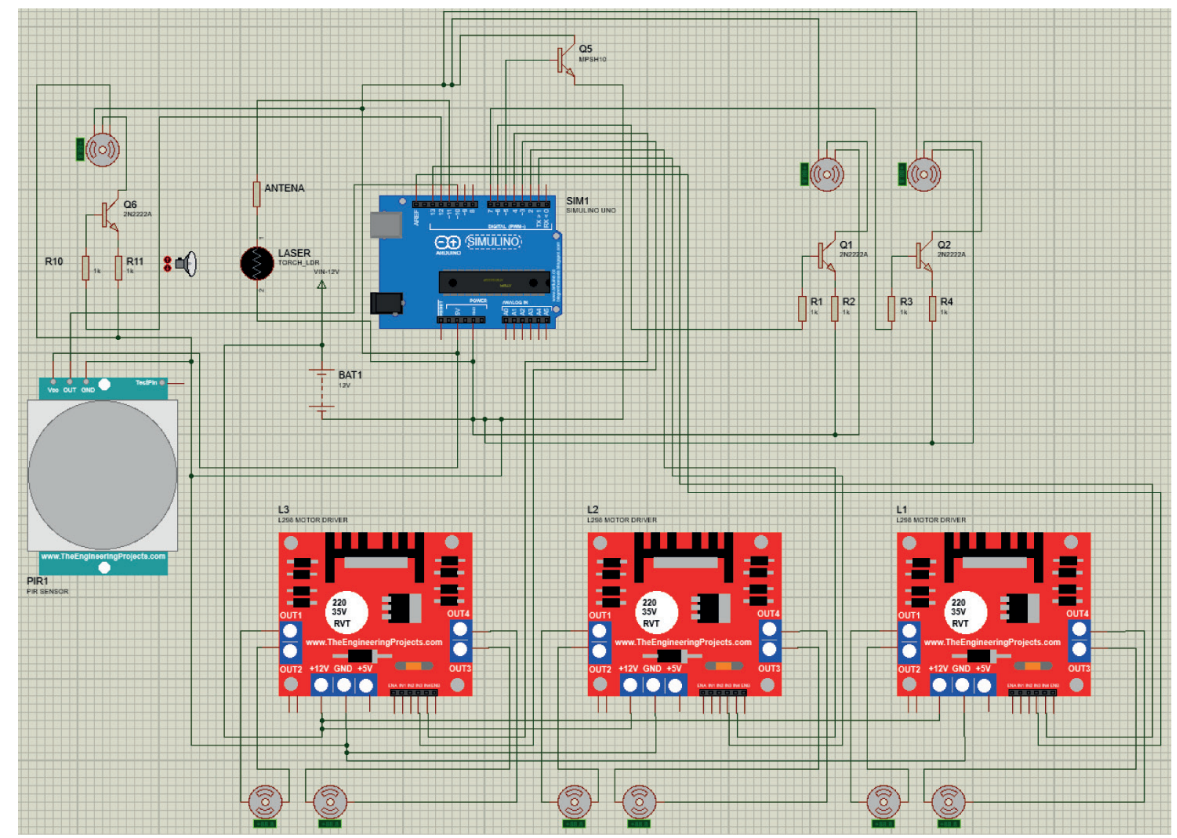

Figura 3. Circuito electrónico del robot diseñado en Proteus. Elaboración propia.

Mediante la figura 4, se presenta la tarjeta electrónica (PCB) simulada con el visualizador 3D del software Proteus.

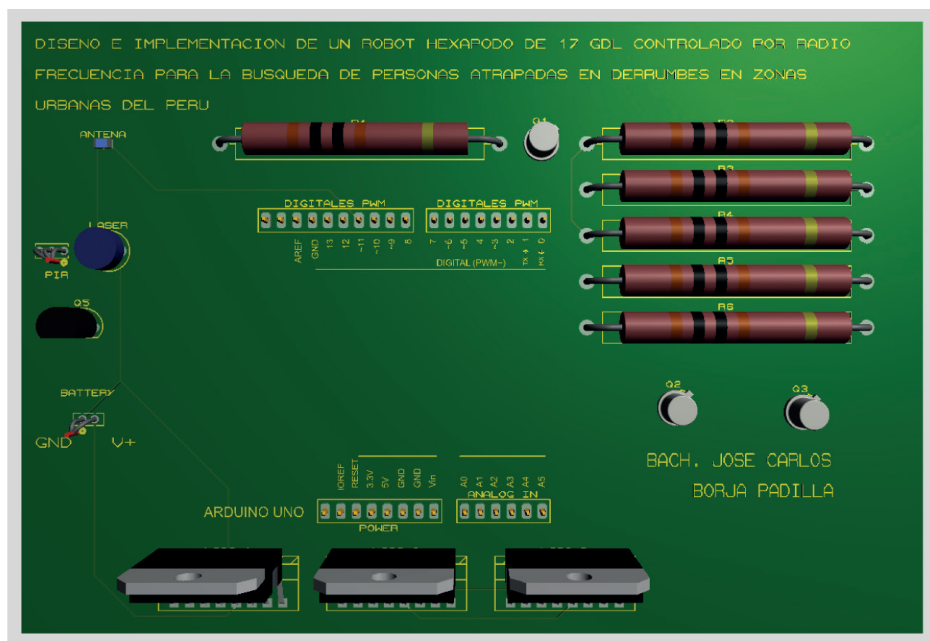

Figura 4. Diseño 3D de la PCB con el software Proteus. Elaboración propia. 


\subsection{Diseño informático}

En la figura 5, se describe en un diagrama de flujo la programación de funcionamiento del robot. Se puede observar el sistema detector de temperatura, de color naranja, encargado de censar la variación de temperatura externa del robot; el sistema locomotor del robot, de color azul, a cargo de los movimientos del robot; y el sistema de verificación, de color verde, que se encarga del sistema visual del robot.

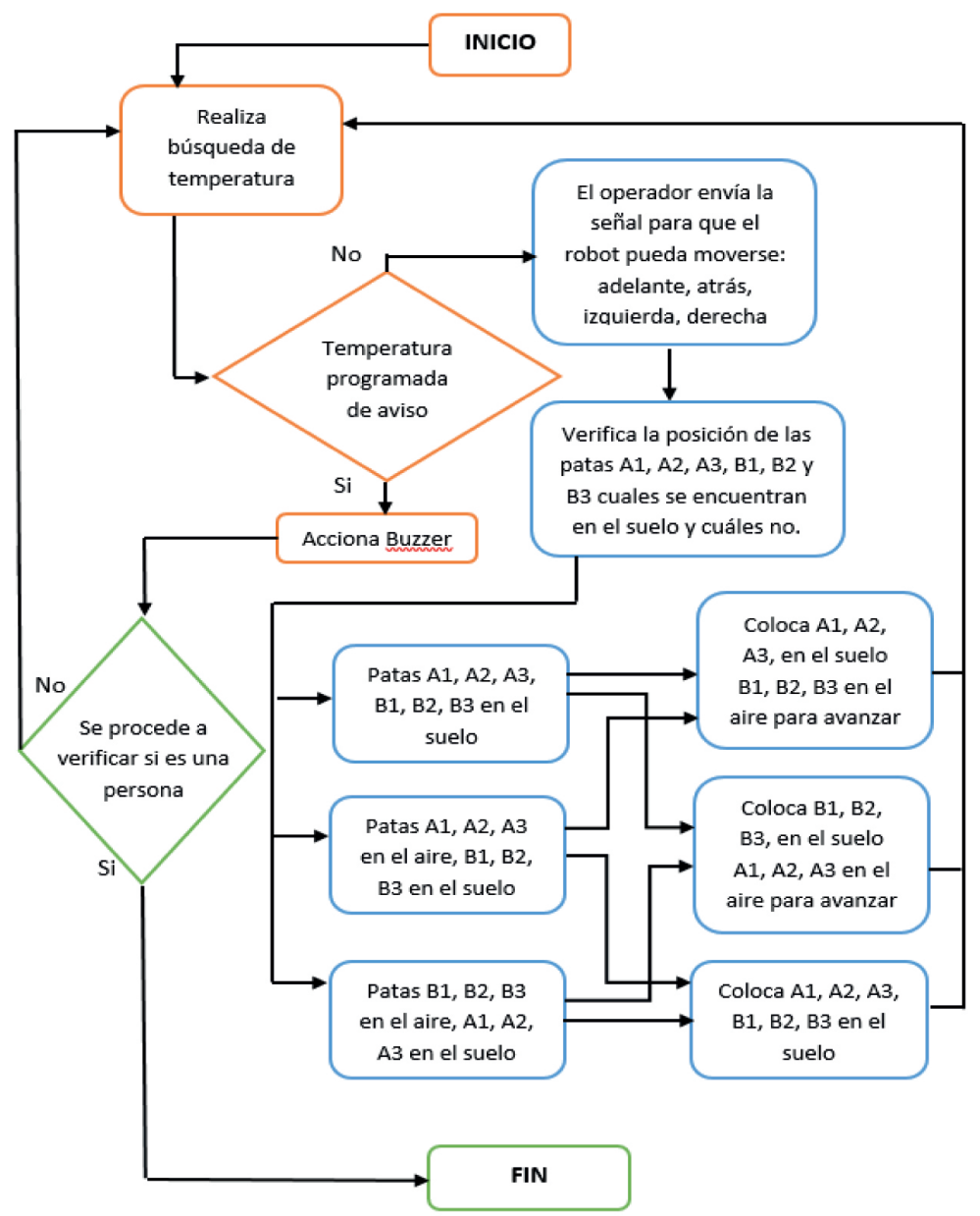

Figura 5. Diagrama de flujo de la programación. Elaboración propia.

\section{IMPLEMENTACIÓN DEL ROBOT}

\subsection{Implementación mecánica}

Se implementó la estructura mecánica del robot y se procedió a realizar el ensamblaje de los brazos y la cabeza al cuerpo del robot, tal como se muestra en la figura 6. 


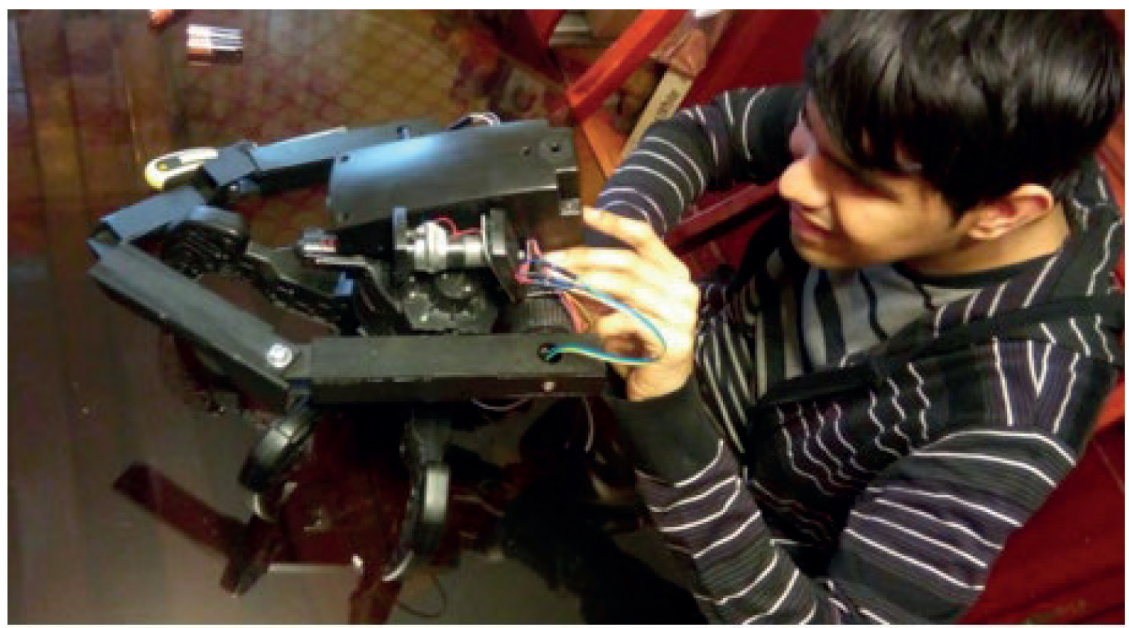

Figura 6. Diagrama de flujo de la programación del robot. Elaboración propia.

\subsection{Implementación electrónica}

Se ensambló la tarjeta electrónica (PCB) y el controlador Arduino Uno en la parte superior de la estructura del robot. Luego se realizó las conexiones eléctricas con las patas. Finalmente, se procedió a instalar la cabeza del robot para proteger el controlador y la tarjeta PCB como se muestra en la figura 7.

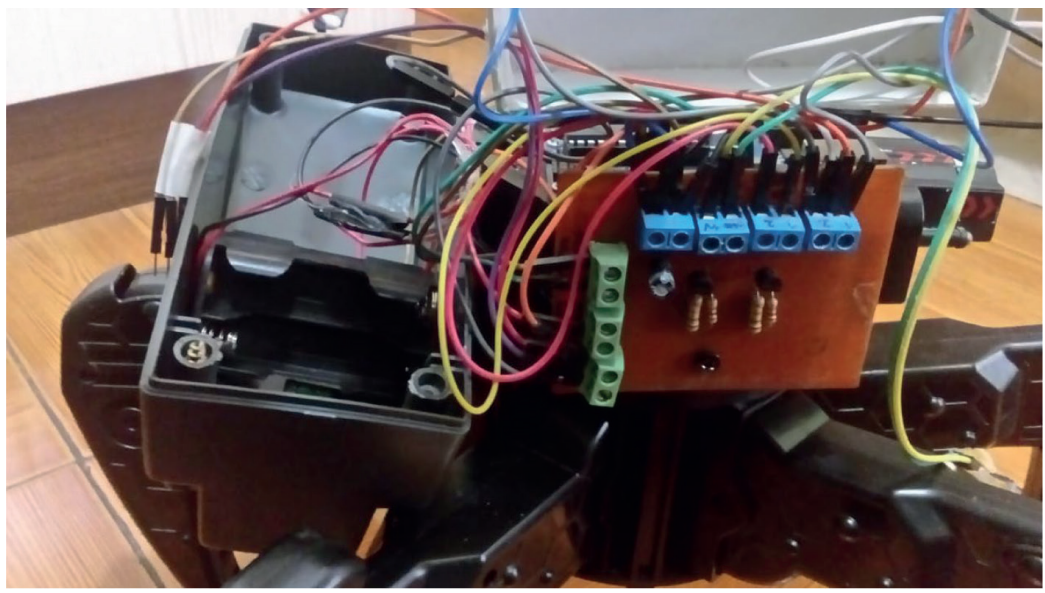

Figura 7. Implementación de tarjeta electrónica. Elaboración propia.

\section{PRUEBAS Y RESULTADOS}

\section{Prueba 1}

La prueba consistió en analizar la estabilidad del robot sobre terrenos accidentados con presencia de escombros [8]. Se obtuvo un resultado favorable, que consistió en una mayor estabilidad, lo que permitió su desplazamiento en todas direcciones sin presentar volcadura ni complicaciones en la relación a su peso, estabilidad y posición de su centro de gravedad, lo que se aprecia en la figura 8. 


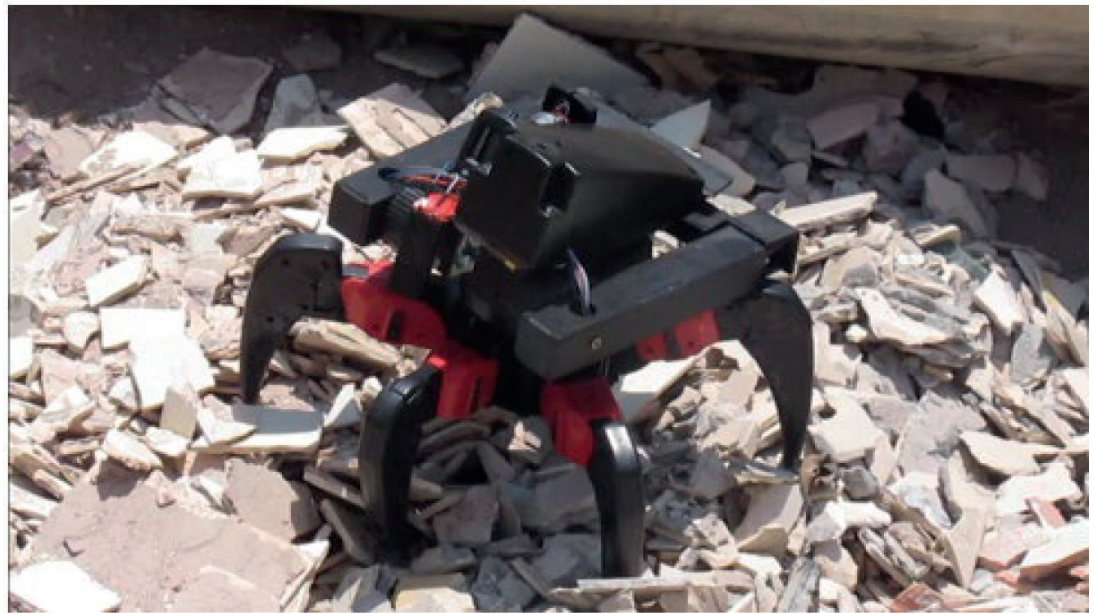

Figura 8. Prueba de estabilidad del robot. Elaboración propia.

\section{Prueba 2}

La segunda prueba consistió en probar el sistema encargado de detectar la temperatura en la palma de la mano de una persona, mediante un dispositivo láser. El resultado obtenido entregó una temperatura en el rango entre $36.3-37.1^{\circ} \mathrm{C}$, lo que indica que la temperatura corporal de la persona censada es normal, tal como se muestra en la figura 9.

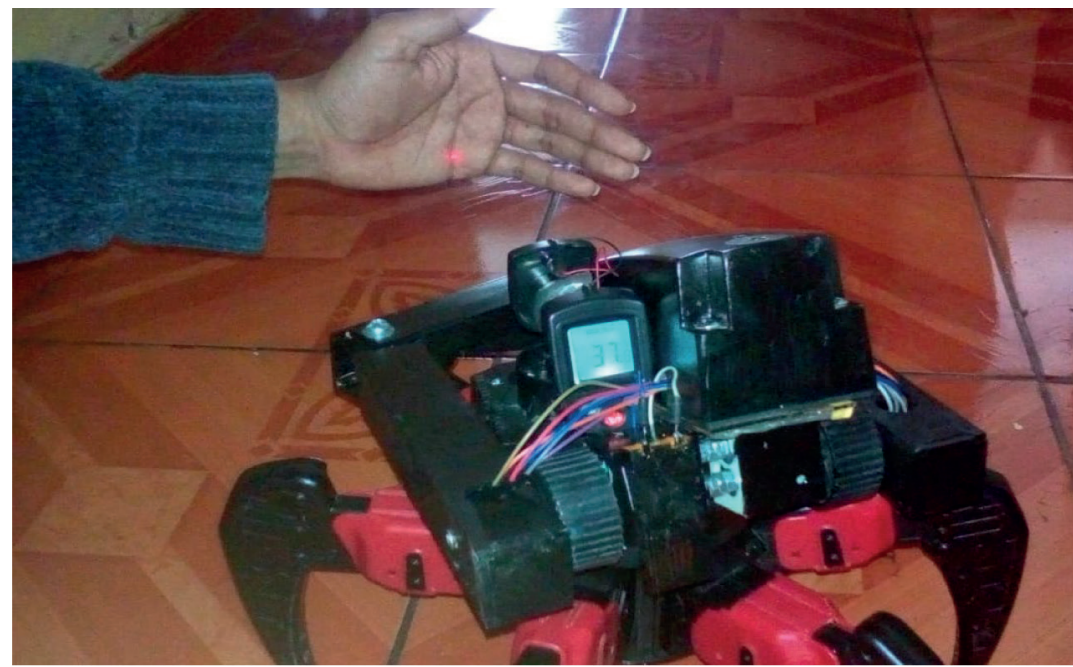

Figura 9. Prueba del sistema de detección de temperatura. Elaboración propia.

\section{Prueba 3}

La tercera prueba consistió en ubicar al robot en un reciente derrumbe y realizar una búsqueda de variación de temperaturas de personas atrapadas. Se obtuvo como resultado la adecuada destreza del robot para desplazarse de manera constante sobre una superficie empinada y con presencia de rocas, durante la búsqueda y censado de la temperatura de las personas. En la figura 10, se aprecia el desplazamiento antes mencionado. 


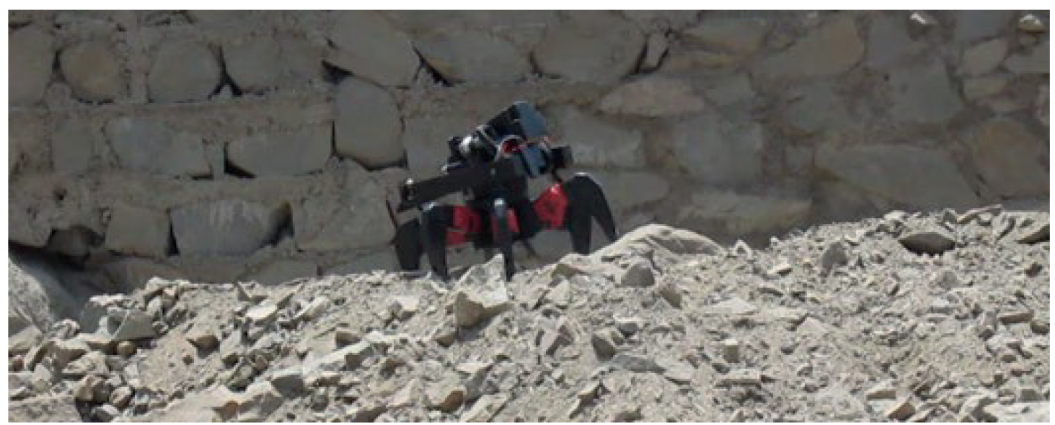

Figura 10. Robot hexápodo realizando búsqueda de personas. Elaboración propia.

\section{Prueba 4}

La prueba final consistió en colocar al robot en un espacio cerrado a modo de simulación de un derrumbe por efecto sísmico y proceder a realizar la búsqueda de personas atrapadas. Se demostró que el robot era capaz de desplazarse entre escombros, adaptándose a condiciones determinadas como la falta de luz, a través de su sistema de iluminación, y el correcto funcionamiento del sistema que detecta la temperatura, al ubicar una persona inconsciente, permitiendo verificar a través de su temperatura corporal que aún se mantiene con vida, tal como se muestra en la figura 11. Así, el rescatista procede a la declaración de una zona segura para iniciar las maniobras de rescate.

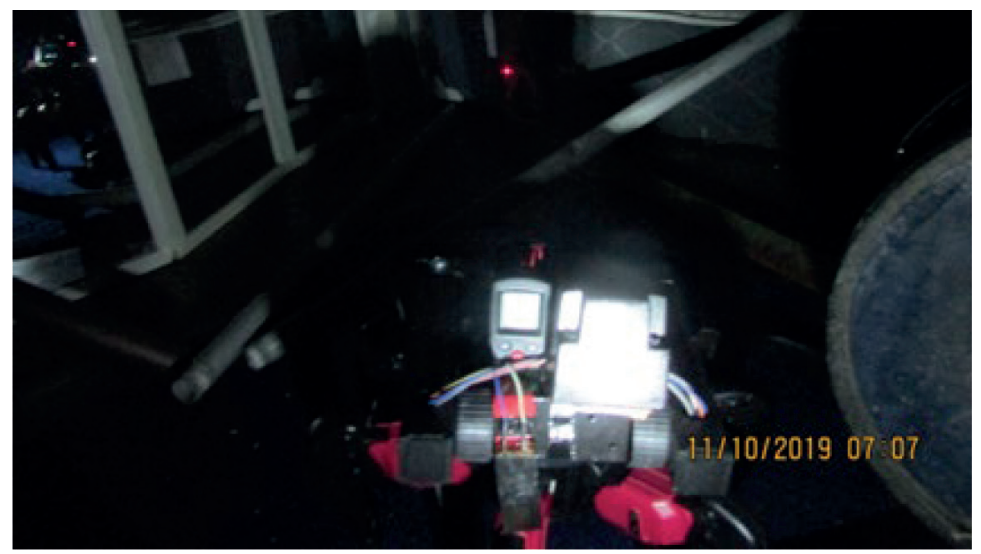

Figura 11. Robot hexápodo realizando búsqueda de personas. Elaboración propia.

\section{CONCLUSIONES}

Concluida la investigación se puede afirmar que, mediante la prueba 1, el robot hexápodo fue capaz de desplazarse con estabilidad por terrenos accidentados, evitando obstáculos de mayor tamaño y apartando obstáculos de menor tamaño. Con la prueba 2, se puede afirmar que el sistema de detección de variación de temperaturas del robot funcionó correctamente, al obtener resultados precisos a menores distancias entre el objetivo y el robot. Con las pruebas 3 y 4 , se logró demostrar que el robot fue capaz de adaptarse a escenarios de derrumbe, rural y urbano, localizar personas atrapadas mediante el censado de temperatura y permitir el inicio seguro del rescate. Para futuras investigaciones, se recomienda incluir un sistema autónomo de navegación y el uso de tecnologías IoT [9]. 


\section{REFERENCIAS}

[1] Instituto Nacional de Defensa Civil, "Compendio Estadístico del INDECI 2017 - Gestión reactiva”, Instituto Nacional de Defensa Civil - INDECI, Lima, Perú, 2017 [En línea]. Disponible en: https://bit.ly/3qytM14 [Accedido: 28-dic-2020].

[2] G. Bermúdez, K. Solange y W. Infante, "La robótica en actividades de búsqueda y rescate urbano", Tecnura, Vol. 8, no. 15, pp. 97-108, 2004 [En línea]. doi: https://doi. org/10.14483/22487638.6165 [Accedido: 28-dic-2020].

[3] A. Ollero, Robótica Manipuladores y robots móviles, Barcelona, España: Marcombo, 2001.

[4] A. Sánchez y C. López, "Artists, Diseño de un robot hexápodo - hardware y software de control", memoria del proyecto, Universidad Politécnica de Vilanova, 2003 [En línea]. Disponible en: https://bit.ly/33TTxkM [Accedido: 28-dic-2020].

[5] A. Barrientos, C. Balaguer y L. Penin, Fundamentos de Robótica, Madrid, España: McGraw-Hill, 2007.

[6] T. Iglesias y E. Bernd, "Aceros Austéniticos al Manganeso: Breve reseña historica”, Remetallica, no. 10, pp. 9-14, 2004 [En línea]. Disponible en: https://bit.ly/3z5z4VD [Accedido: 28-dic-2020].

[7] E. García, Compilador C CCS y simulador proteus para micro controladores pics, Ciudad de México, Mexico: Marcombo, 2008.

[8] J. Suárez, Deslizamientos, Tomo 1: Análisis Geotécnico, Bucaramanga, Colombia: Geotecnologia, 2009.

[9] C. Muñoz, R. Castro, R. Palomares y J. Cornejo, "Mechatronics Development of Terrestrial Mobile Robot for Exploring and Monitoring Environmental Parameters at Mine Analogue Sites using IoT Platform”, 2020 IEEE XXVII International Conference on Electronics, Electrical Engineering and Computing (INTERCON), Lima, Perú, pp. 1-4, [En línea]. doi: 10.1109/ INTERCON50315.2020.9220227 [Accedido: 28-dic-2020] 